Acta Crystallographica Section A

Foundations of

Crystallography

ISSN 0108-7673

Received 5 January 2006

Accepted 16 February 2006

(C) 2006 International Union of Crystallography

Printed in Great Britain - all rights reserved

\section{Spectral decomposition of the linear elastic tensor for trigonal symmetry; classification of symmetry restrictions for arbitrary point groups}

\author{
Hans Grimmer \\ Laboratory for Development and Methods, Condensed Matter Research with Neutrons and Muons, \\ Paul Scherrer Institut, CH-5232 Villigen PSI, Switzerland. Correspondence e-mail: \\ hans.grimmer@psi.ch
}

\begin{abstract}
The linear compliance tensor for trigonal symmetry has four different eigenvalues, two of which have multiplicity 1 , the others multiplicity 2 . They and the corresponding eigenvectors have been calculated in terms of the seven parameters of the corresponding Voigt matrix. Necessary and sufficient conditions have been derived for these components to guarantee positive eigenvalues and thus a positive strain energy. The hierarchy of restrictions on the linear elastic tensors that follow from Neumann's principle for arbitrary point groups in three dimensions has been established for the standard choice of the Cartesian coordinate system, as well as in coordinate-independent form.
\end{abstract}

\section{Introduction}

In a series of papers, Theocaris \& Sokolis $(1999,2000 a, b, 2001)$ described the spectral decomposition of the elastic compliance and stiffness fourth-rank tensors for crystals with different point-group symmetries. Their approach is based on the work of Rychlewski $(1984 a, b)$, who set the general stage for the spectral decomposition of the elastic tensors and considered elastically isotropic and transversely isotropic media as examples. Theocaris \& Sokolis $(1999,2000 a)$ found that the number of different eigenvalues plus the number of different 'eigenangles' is equal to the number of independent tensor components for monoclinic, orthorhombic, tetragonal, hexagonal and cubic crystals as well as in the isotropic case. For trigonal crystals, they found a result that did not fit into the general picture sketched above (Theocaris \& Sokolis, 2000b). In Theocaris \& Sokolis (2001), they rediscussed the results obtained in Theocaris \& Sokolis $(1999,2000 a)$, but did not mention the trigonal case, maybe because they felt that something was wrong in Theocaris \& Sokolis $(2000 b)$. We, therefore, reconsider the trigonal case in the present paper.

The compliance and stiffness tensors being invariant under inversion, $\overline{1}$, the restrictions on their form imposed by pointgroup symmetry will depend only on the Laue class. Because there are 11 Laue classes, the number of different restrictions cannot be larger than 12 if the isotropic case is also included. It is well known that the restrictions are the same for the two hexagonal Laue classes and also for the two cubic Laue classes (see e.g. Nye, 1985; Landolt-Börnstein, 1979; Authier \& Zarembovitch, 2003). It will be shown that considering also non-crystallographic point groups does not lead to other restrictions. Forte \& Vianello (1996) distinguish only eight symmetry classes of elastic tensors, not ten. It will be shown that this is because the tensors of the two trigonal Laue classes belong to the same symmetry class; similarly, the tensors of the two tetragonal Laue classes.

\section{Descriptions of linear elasticity}

Hooke's law of linear elasticity can be generalized as follows to anisotropic media (see e.g. Nye, 1985; Landolt-Börnstein, 1979; Theocaris \& Sokolis, 1999; Authier \& Zarembovitch, 2003):

$$
\varepsilon_{i j}=S_{i j k l} \sigma_{k l},
$$

where the indices run from 1 to 3 and summation over repeated indices is implied. The second-rank tensors $\sigma_{k l}$ and $\varepsilon_{i j}$ describe stress and strain, respectively; they are symmetric: $\sigma_{k l}=\sigma_{l k}$ and $\varepsilon_{k l}=\varepsilon_{l k}$. The fourth-rank tensor $S_{i j k l}$ describes compliance and has the following symmetries: $S_{i j k l}=S_{j i k l}=$ $S_{i j l k}=S_{k l i j}$. Owing to these symmetries, (1) can be expressed in matrix form as follows:

$\left[\begin{array}{c}\varepsilon_{11} \\ \varepsilon_{22} \\ \varepsilon_{33} \\ 2 \varepsilon_{23} \\ 2 \varepsilon_{13} \\ 2 \varepsilon_{12}\end{array}\right]=\left[\begin{array}{cccccc}S_{1111} & S_{1122} & S_{1133} & 2 S_{1123} & 2 S_{1113} & 2 S_{1112} \\ S_{1122} & S_{2222} & S_{2233} & 2 S_{2223} & 2 S_{2213} & 2 S_{2212} \\ S_{1133} & S_{2233} & S_{3333} & 2 S_{3323} & 2 S_{3313} & 2 S_{3312} \\ 2 S_{1123} & 2 S_{2223} & 2 S_{3323} & 4 S_{2323} & 4 S_{2313} & 4 S_{2312} \\ 2 S_{1113} & 2 S_{2213} & 2 S_{3313} & 4 S_{2313} & 4 S_{1313} & 4 S_{1312} \\ 2 S_{1112} & 2 S_{2212} & 2 S_{3312} & 4 S_{2312} & 4 S_{1312} & 4 S_{1212}\end{array}\right]\left[\begin{array}{c}\sigma_{11} \\ \sigma_{22} \\ \sigma_{33} \\ \sigma_{23} \\ \sigma_{13} \\ \sigma_{12}\end{array}\right]$,




$$
\left[\begin{array}{l}
\varepsilon_{1} \\
\varepsilon_{2} \\
\varepsilon_{3} \\
\varepsilon_{4} \\
\varepsilon_{5} \\
\varepsilon_{6}
\end{array}\right]=\left[\begin{array}{llllll}
s_{11} & s_{12} & s_{13} & s_{14} & s_{15} & s_{16} \\
s_{12} & s_{22} & s_{23} & s_{24} & s_{25} & s_{26} \\
s_{13} & s_{23} & s_{33} & s_{34} & s_{35} & s_{36} \\
s_{14} & s_{24} & s_{34} & s_{44} & s_{45} & s_{46} \\
s_{15} & s_{25} & s_{35} & s_{45} & s_{55} & s_{56} \\
s_{16} & s_{26} & s_{36} & s_{46} & s_{56} & s_{66}
\end{array}\right]\left[\begin{array}{l}
\sigma_{1} \\
\sigma_{2} \\
\sigma_{3} \\
\sigma_{4} \\
\sigma_{5} \\
\sigma_{6}
\end{array}\right]
$$

or shortly

$$
\varepsilon_{\mu}=s_{\mu \nu} \sigma_{v},
$$

where the Greek indices run from 1 to 6 .

Theocaris \& Sokolis (1999) consider instead of the matrix $s_{\mu \nu}$ a matrix in which the components without indices 4,5 or 6 are the same, those with one index 4,5 or 6 are $2^{1 / 2}$ times smaller and those with both indices in the range 4 to 6 are 2 times smaller. It follows that

$$
\begin{aligned}
& {\left[\begin{array}{c}
\varepsilon_{1} \\
\varepsilon_{2} \\
\varepsilon_{3} \\
2^{-1 / 2} \varepsilon_{4} \\
2^{-1 / 2} \varepsilon_{5} \\
2^{-1 / 2} \varepsilon_{6}
\end{array}\right]} \\
& =\left[\begin{array}{cccccc}
s_{11} & s_{12} & s_{13} & 2^{-1 / 2} s_{14} & 2^{-1 / 2} s_{15} & 2^{-1 / 2} s_{16} \\
s_{12} & s_{22} & s_{23} & 2^{-1 / 2} s_{24} & 2^{-1 / 2} s_{25} & 2^{-1 / 2} s_{26} \\
s_{13} & s_{23} & s_{33} & 2^{-1 / 2} s_{34} & 2^{-1 / 2} s_{35} & 2^{-1 / 2} s_{36} \\
2^{-1 / 2} s_{14} & 2^{-1 / 2} s_{24} & 2^{-1 / 2} s_{34} & 2^{-1} s_{44} & 2^{-1} s_{45} & 2^{-1} s_{46} \\
2^{-1 / 2} s_{15} & 2^{-1 / 2} s_{25} & 2^{-1 / 2} s_{35} & 2^{-1} s_{45} & 2^{-1} s_{55} & 2^{-1} s_{56} \\
2^{-1 / 2} s_{16} & 2^{-1 / 2} s_{26} & 2^{-1 / 2} s_{36} & 2^{-1} s_{46} & 2^{-1} s_{56} & 2^{-1} s_{66}
\end{array}\right]\left[\begin{array}{c}
\sigma_{1} \\
\sigma_{2} \\
\sigma_{3} \\
2^{1 / 2} \sigma_{4} \\
2^{1 / 2} \sigma_{5} \\
2^{1 / 2} \sigma_{6}
\end{array}\right]
\end{aligned}
$$

or

$$
\begin{aligned}
{\left[\begin{array}{c}
\varepsilon_{11} \\
\varepsilon_{22} \\
\varepsilon_{33} \\
2^{1 / 2} \varepsilon_{23} \\
2^{1 / 2} \varepsilon_{13} \\
2^{1 / 2} \varepsilon_{12}
\end{array}\right] } \\
=\left[\begin{array}{cccccc}
S_{1111} & S_{1122} & S_{1133} & 2^{1 / 2} S_{1123} & 2^{1 / 2} S_{1113} & 2^{1 / 2} S_{1112} \\
S_{1122} & S_{2222} & S_{2233} & 2^{1 / 2} S_{2223} & 2^{1 / 2} S_{2213} & 2^{1 / 2} S_{2212} \\
S_{1133} & S_{2233} & S_{3333} & 2^{1 / 2} S_{3323} & 2^{1 / 2} S_{3313} & 2^{1 / 2} S_{3312} \\
2^{1 / 2} S_{1123} & 2^{1 / 2} S_{2223} & 2^{1 / 2} S_{3323} & 2 S_{2323} & 2 S_{2313} & 2 S_{2312} \\
2^{1 / 2} S_{1113} & 2^{1 / 2} S_{2213} & 2^{1 / 2} S_{3313} & 2 S_{2313} & 2 S_{1313} & 2 S_{1312} \\
2^{1 / 2} S_{1112} & 2^{1 / 2} S_{2212} & 2^{1 / 2} S_{3312} & 2 S_{2312} & 2 S_{1312} & 2 S_{1212}
\end{array}\right]\left[\begin{array}{c}
\sigma_{11} \\
\sigma_{22} \\
\sigma_{33} \\
2^{1 / 2} \sigma_{23} \\
2^{1 / 2} \sigma_{13} \\
2^{1 / 2} \sigma_{12}
\end{array}\right] .
\end{aligned}
$$

Conversely, the stresses can be expressed by the strains,

$$
\sigma_{i j}=C_{i j k l} \varepsilon_{k l}
$$

where $C_{i j k l}$ describes stiffness and has the following symmetries: $C_{i j k l}=C_{j i k l}=C_{i j l k}=C_{k l i j}$. Owing to these symmetries, (7) can be expressed in matrix form as follows:

$\left[\begin{array}{l}\sigma_{11} \\ \sigma_{22} \\ \sigma_{33} \\ \sigma_{23} \\ \sigma_{13} \\ \sigma_{12}\end{array}\right]=\left[\begin{array}{llllll}C_{1111} & C_{1122} & C_{1133} & C_{1123} & C_{1113} & C_{1112} \\ C_{1122} & C_{2222} & C_{2233} & C_{2223} & C_{2213} & C_{2212} \\ C_{1133} & C_{2233} & C_{3333} & C_{3323} & C_{3313} & C_{3312} \\ C_{1123} & C_{2223} & C_{3323} & C_{2323} & C_{2313} & C_{2312} \\ C_{1113} & C_{2213} & C_{3313} & C_{2313} & C_{1313} & C_{1312} \\ C_{1112} & C_{2212} & C_{3312} & C_{2312} & C_{1312} & C_{1212}\end{array}\right]\left[\begin{array}{c}\varepsilon_{11} \\ \varepsilon_{22} \\ \varepsilon_{33} \\ 2 \varepsilon_{23} \\ 2 \varepsilon_{13} \\ 2 \varepsilon_{12}\end{array}\right]$,

(8) which in Voigt notation becomes

$$
\left[\begin{array}{l}
\sigma_{1} \\
\sigma_{2} \\
\sigma_{3} \\
\sigma_{4} \\
\sigma_{5} \\
\sigma_{6}
\end{array}\right]=\left[\begin{array}{llllll}
c_{11} & c_{12} & c_{13} & c_{14} & c_{15} & c_{16} \\
c_{12} & c_{22} & c_{23} & c_{24} & c_{25} & c_{26} \\
c_{13} & c_{23} & c_{33} & c_{34} & c_{35} & c_{36} \\
c_{14} & c_{24} & c_{34} & c_{44} & c_{45} & c_{46} \\
c_{15} & c_{25} & c_{35} & c_{45} & c_{55} & c_{56} \\
c_{16} & c_{26} & c_{36} & c_{46} & c_{56} & c_{66}
\end{array}\right]\left[\begin{array}{l}
\varepsilon_{1} \\
\varepsilon_{2} \\
\varepsilon_{3} \\
\varepsilon_{4} \\
\varepsilon_{5} \\
\varepsilon_{6}
\end{array}\right]
$$

or shortly

$$
\sigma_{\mu}=c_{\mu \nu} \varepsilon_{\nu}
$$

Extending the approach of Theocaris \& Sokolis (1999) also to stiffness, we consider instead of the matrix $c_{\mu \nu}$ a matrix in which the components without indices 4,5 or 6 are the same, those with one index 4,5 or 6 are $2^{1 / 2}$ times larger and those with both indices in the range 4 to 6 are 2 times larger. It follows that

$\left[\begin{array}{c}\sigma_{1} \\ \sigma_{2} \\ \sigma_{3} \\ 2^{1 / 2} \sigma_{4} \\ 2^{1 / 2} \sigma_{5} \\ 2^{1 / 2} \sigma_{6}\end{array}\right]$
$=\left[\begin{array}{cccccc}c_{11} & c_{12} & c_{13} & 2^{1 / 2} c_{14} & 2^{1 / 2} c_{15} & 2^{1 / 2} c_{16} \\ c_{12} & c_{22} & c_{23} & 2^{1 / 2} c_{24} & 2^{1 / 2} c_{25} & 2^{1 / 2} c_{26} \\ c_{13} & c_{23} & c_{33} & 2^{1 / 2} c_{34} & 2^{1 / 2} c_{35} & 2^{1 / 2} c_{36} \\ 2^{1 / 2} c_{14} & 2^{1 / 2} c_{24} & 2^{1 / 2} c_{34} & 2 c_{44} & 2 c_{45} & 2 c_{46} \\ 2^{1 / 2} c_{15} & 2^{1 / 2} c_{25} & 2^{1 / 2} c_{35} & 2 c_{45} & 2 c_{55} & 2 c_{56} \\ 2^{1 / 2} c_{16} & 2^{1 / 2} c_{26} & 2^{1 / 2} c_{36} & 2 c_{46} & 2 c_{56} & 2 c_{66}\end{array}\right]\left[\begin{array}{c}\varepsilon_{1} \\ \varepsilon_{2} \\ \varepsilon_{3} \\ 2^{-1 / 2} \varepsilon_{4} \\ 2^{-1 / 2} \varepsilon_{5} \\ 2^{-1 / 2} \varepsilon_{6}\end{array}\right]$

or

$$
\begin{gathered}
{\left[\begin{array}{c}
\sigma_{11} \\
\sigma_{22} \\
\sigma_{33} \\
2^{1 / 2} \sigma_{23} \\
2^{1 / 2} \sigma_{13} \\
2^{1 / 2} \sigma_{12}
\end{array}\right]} \\
=\left[\begin{array}{cccccc}
C_{1111} & C_{1122} & C_{1133} & 2^{1 / 2} C_{1123} & 2^{1 / 2} C_{1113} & 2^{1 / 2} C_{1112} \\
C_{1122} & C_{2222} & C_{2233} & 2^{1 / 2} C_{2223} & 2^{1 / 2} C_{2213} & 2^{1 / 2} C_{2212} \\
C_{1133} & C_{2233} & C_{3333} & 2^{1 / 2} C_{3323} & 2^{1 / 2} C_{3313} & 2^{1 / 2} C_{3312} \\
2^{1 / 2} C_{1123} & 2^{1 / 2} C_{2223} & 2^{1 / 2} C_{3323} & 2 C_{2323} & 2 C_{2313} & 2 C_{2312} \\
2^{1 / 2} C_{1113} & 2^{1 / 2} C_{2213} & 2^{1 / 2} C_{3313} & 2 C_{2313} & 2 C_{1313} & 2 C_{1312} \\
2^{1 / 2} C_{1112} & 2^{1 / 2} C_{2212} & 2^{1 / 2} C_{3312} & 2 C_{2312} & 2 C_{1312} & 2 C_{1212}
\end{array}\right]\left[\begin{array}{c}
\varepsilon_{11} \\
\varepsilon_{22} \\
\varepsilon_{33} \\
2^{1 / 2} \varepsilon_{23} \\
2^{1 / 2} \varepsilon_{13} \\
2^{1 / 2} \varepsilon_{12}
\end{array}\right]
\end{gathered}
$$

Equations (6) and (12) show that in this alternative approach the numerical factors appearing when changing from tensor to matrix notation are distributed equally on stress and strain, and equally on compliance and stiffness.

Let me explain the reason for considering the matrices appearing in (5) and (11) rather than those in (3) and (9). The identity map in the space of symmetric tensors of second rank is given by

$$
I_{i j k l}=\frac{1}{2}\left(\delta_{i k} \delta_{j l}+\delta_{i l} \delta_{j k}\right)
$$


Indeed, $I_{i j k l} \varepsilon_{k l} \equiv \frac{1}{2}\left(\varepsilon_{k l}+\varepsilon_{l k}\right)=\varepsilon_{k l}$. The non-zero elements of $I_{i j k l}$ are $I_{1111}=I_{2222}=I_{3333}=1$ and $I_{2323}\left(=I_{3223}=I_{2332}=I_{3232}\right)=$ $I_{1313}\left(=I_{3113}=\ldots\right)=I_{1212}\left(=I_{2112}=\ldots\right)=\frac{1}{2}$. Going over to $6 \times 6$ matrix notation, we therefore obtain the identity matrix $I_{\mu \nu}=\delta_{\mu \nu}$ if we associate powers of 2 with the elements $I_{i j k l}$ as in equations (6), (12), but not if we do it as in equations (2), (8). The identity matrix has all its six eigenvalues equal to 1 , as expected for the identity map in the six-dimensional space of symmetric tensors of second rank. We conclude that the eigenvalues of the matrices appearing in equations (5), (6), (11), (12) have to be considered in the spectral decomposition of the compliance and stiffness tensor, not the eigenvalues of the matrices appearing in equations (2), (3), (8), (9).

\section{Eigenvalues and eigenvectors of the matrices describing the elastic properties for trigonal symmetry}

The compliance of a crystal is usually characterized by specifying the components $s_{\mu \nu}$ of the matrix appearing in (3), not more than 21 of which can be independent because the matrix is symmetric. For crystals with higher than triclinic point-group symmetry, the number of independent components is reduced by Neumann's principle. For the trigonal crystal classes it is 7 for 3 and $\overline{3} ; 6$ for $32,3 m$ and $\overline{3} m$. These two cases will be referred to as trigonal-7 and trigonal- 6 , respectively. The form of the matrices appearing in (3) and (9) depends only on the Laue class and has been given e.g. in Table 9 of Nye (1985) for the 11 crystallographic Laue classes and for isotropic media. It follows from that table that, for trigonal symmetry, the matrix in (5) has the form

$\left[\begin{array}{cccccc}s_{11} & s_{12} & s_{13} & 2^{-1 / 2} s_{14} & 2^{-1 / 2} s_{15} & 0 \\ s_{12} & s_{11} & s_{13} & -2^{-1 / 2} s_{14} & -2^{-1 / 2} s_{15} & 0 \\ s_{13} & s_{13} & s_{33} & 0 & 0 & 0 \\ 2^{-1 / 2} s_{14} & -2^{-1 / 2} s_{14} & 0 & 2^{-1} s_{44} & 0 & -s_{15} \\ 2^{-1 / 2} s_{15} & -2^{-1 / 2} s_{15} & 0 & 0 & 2^{-1} s_{44} & s_{14} \\ 0 & 0 & 0 & -s_{15} & s_{14} & s_{11}-s_{12}\end{array}\right]$

if the $x_{3}$ axis of the Cartesian coordinate system is chosen along the principal symmetry axis. In the case of $32,3 \mathrm{~m}$ and $\overline{3} \mathrm{~m}$, we have $s_{15}=0$ if $x_{1}$ is chosen along a secondary symmetry axis. The corresponding stiffness matrix is simply obtained by replacing $s$ by $c$. This is in contrast to the matrices $s_{\mu \nu}$ and $c_{\mu \nu}$ considered by Nye (1985), where $s_{66}=2\left(s_{11}-s_{12}\right)$ and $c_{66}=$ $\frac{1}{2}\left(c_{11}-c_{12}\right)$.

Notice that (14) does not agree with the matrix for which Theocaris \& Sokolis (2000b) calculate the eigenvalues in their equation (7). As a consequence, our results for the eigenvalues and eigenangles will not agree with their equations (8) and (14), respectively.

Matrix (14) has the eigenvalues

$$
\lambda_{1,2}=\frac{s_{11}+s_{12}+s_{33}}{2} \pm\left[\left(\frac{s_{11}+s_{12}-s_{33}}{2}\right)^{2}+2 s_{13}^{2}\right]^{1 / 2}
$$

and $\lambda_{3,4}=\frac{s_{11}-s_{12}+\frac{1}{2} s_{44}}{2} \pm\left[\left(\frac{s_{11}-s_{12}-\frac{1}{2} s_{44}}{2}\right)^{2}+s_{14}^{2}+s_{15}^{2}\right]^{1 / 2}$

where the plus sign holds for $\lambda_{1}$ and $\lambda_{3}$, the minus sign for $\lambda_{2}$ and $\lambda_{4}$. The multiplicity is 1 for $\lambda_{1}$ and $\lambda_{2}, 2$ for $\lambda_{3}$ and $\lambda_{4}$.

The corresponding eigenvectors are

$\overline{\sigma_{1}}=\left\{-2^{-1 / 2}\left(\sigma_{1}+\sigma_{2}\right) \sin \omega+\sigma_{3} \cos \omega\right\}$

$\times\left[-2^{-1 / 2} \sin \omega,-2^{-1 / 2} \sin \omega, \cos \omega, 0,0,0\right]^{T}$

$\overline{\sigma_{2}}=\left\{2^{-1 / 2}\left(\sigma_{1}+\sigma_{2}\right) \cos \omega+\sigma_{3} \sin \omega\right\}$

$$
\times\left[2^{-1 / 2} \cos \omega, 2^{-1 / 2} \cos \omega, \sin \omega, 0,0,0\right]^{T}
$$

$\overline{\sigma_{3}}=2^{1 / 2}\left\{-\frac{1}{2}\left(\sigma_{1}-\sigma_{2}\right) \sin \xi+\left(\sigma_{4} \cos \rho+\sigma_{5} \sin \rho\right) \cos \xi\right\}$

$\times\left[-2^{-1 / 2} \sin \xi, 2^{-1 / 2} \sin \xi, 0, \cos \xi \cos \rho, \cos \xi \sin \rho, 0\right]^{T}$

$\overline{\sigma_{4}}=2^{1 / 2}\left\{\frac{1}{2}\left(\sigma_{1}-\sigma_{2}\right) \cos \xi+\left(\sigma_{4} \cos \rho+\sigma_{5} \sin \rho\right) \sin \xi\right\}$

$\times\left[2^{-1 / 2} \cos \xi,-2^{-1 / 2} \cos \xi, 0, \sin \xi \cos \rho, \sin \xi \sin \rho, 0\right]^{T}$

$\overline{\sigma_{5}}=2^{1 / 2}\left\{\left(-\sigma_{4} \sin \rho+\sigma_{5} \cos \rho\right) \cos \xi-\sigma_{6} \sin \xi\right\}$

$\times[0,0,0,-\cos \xi \sin \rho, \cos \xi \cos \rho,-\sin \xi]^{T}$

$\overline{\sigma_{6}}=2^{1 / 2}\left\{\left(-\sigma_{4} \sin \rho+\sigma_{5} \cos \rho\right) \sin \xi+\sigma_{6} \cos \xi\right\}$

$\times[0,0,0,-\sin \xi \sin \rho, \sin \xi \cos \rho, \cos \xi]^{T}$,

where these six eigenvectors have been normalized such that

$$
\sum_{i=1}^{6} \overline{\boldsymbol{\sigma}_{i}}=\left[\sigma_{1}, \sigma_{2}, \sigma_{3}, 2^{1 / 2} \sigma_{4}, 2^{1 / 2} \sigma_{5}, 2^{1 / 2} \sigma_{6}\right]^{T} .
$$

Notice that the vectors between brackets in $(16 a)-(16 f)$ have unit length. The vectors $\overline{\sigma_{3}}$ and $\overline{\sigma_{5}}$ belong to the same eigenvalue, and similarly $\overline{\sigma_{4}}$ and $\overline{\sigma_{6}}$. Eigenvectors corresponding to different eigenvalues are always orthogonal. Notice that the vectors have been chosen such that also $\overline{\sigma_{3}} \perp \overline{\sigma_{5}}$ and $\overline{\sigma_{4}} \perp \overline{\sigma_{6}}$.

The angles appearing in expressions (16), called 'eigenangles' are given by

$$
\begin{aligned}
\tan 2 \omega & =\frac{8^{1 / 2} s_{13}}{s_{11}+s_{12}-s_{33}}, \\
\tan 2 \xi & =\frac{2\left(s_{14}^{2}+s_{15}^{2}\right)^{1 / 2}}{s_{11}-s_{12}-\frac{1}{2} s_{44}}, \\
\tan \rho & =\frac{s_{15}}{s_{14}} .
\end{aligned}
$$

Notice that (18a) does not uniquely determine $\sin \omega$ and $\cos \omega$; there are four possible pairs $(\sin \omega, \cos \omega)$ related as $(s, c)$, $(-s,-c),(c,-s),(-c, s)$. Changing from the first to the second choice leaves all $\bar{\sigma}_{i}$ unaffected; changing to the third or fourth choice exchanges $\overline{\sigma_{1}}$ and $\overline{\sigma_{2}}$, which corresponds to exchanging the eigenvectors to the eigenvalues $\lambda_{1}$ and $\lambda_{2}$. The situation is similar with regard to $\xi$; a simultaneous change of the signs of $\sin \xi$ and $\cos \xi$ leaves all $\overline{\sigma_{i}}$ unaffected; the other two possible changes exchange $\overline{\sigma_{3}}$ and $\overline{\sigma_{4}}$, as well as $\overline{\sigma_{5}}$ and $\overline{\sigma_{6}}$, corresponding to an exchange of the eigenvectors to the eigenvalues $\lambda_{3}$ and $\lambda_{4}$. Equation (18c) determines $\sin \rho$ and $\cos \rho$ up to a simultaneous change of sign. Such a change essentially 
affects the vectors $\overline{\boldsymbol{\sigma}_{3}}, \overline{\boldsymbol{\sigma}_{4}}, \overline{\boldsymbol{\sigma}_{5}}$ and $\overline{\boldsymbol{\sigma}_{6}}$; it has to be verified which of the two possibilities corresponds to eigenvectors; see the examples $(a)$ and $(b)$ below.

In the case trigonal-6, we have $s_{15}=0$, i.e. $\rho=0$ or $180^{\circ}$; the sum of the number of independent eigenvalues, i.e. 4 , and the number of independent eigenangles, i.e. 3 or 2, therefore equals the number of independent components $s_{i j}$ also in the two trigonal cases. Table 9 of Nye (1985) shows that the hexagonal form is also obtained by putting $s_{14}=0$, whence $\lambda_{3}=$ $s_{11}-s_{12}, \lambda_{4}=\frac{1}{2} s_{44}$ and $\tan 2 \xi=0$, in agreement with Theocaris \& Sokolis $(2000 a)$.

Let us consider two numerical examples, dolomite of type trigonal-7 and calcite of type trigonal-6.

(a) Dolomite, $\mathrm{CaMg}\left(\mathrm{CO}_{3}\right)_{2}$, has space group $R \overline{3}$. LandoltBörnstein (1984) gives its compliance constants in units of $(\mathrm{TPa})^{-1}$ as $s_{11}=7.04, s_{33}=11.2, s_{44}=31.9, s_{12}=-2.4, s_{13}=$ $-2.3, s_{14}=4.6, s_{15}=-3.3$. These values are based on the results by Humbert $\&$ Plicque (1972), who chose $x_{1}$ along a shortest lattice vector perpendicular to the symmetry axis. Equation (15) then gives for the eigenvalues in $(\mathrm{TPa})^{-1}: \lambda_{1}=12.5, \lambda_{2}=$ $3.30, \lambda_{3}=19.2, \lambda_{4}=6.16$. It has been checked numerically that the solutions $\omega=22.4^{\circ}, \xi=30.1^{\circ}, \rho=144.3^{\circ}$ of (18) give rise to eigenvectors but $\rho=144.3-180=-35.7^{\circ}$ does not. The eigenvectors are

$$
\begin{aligned}
& \overline{\sigma_{1}} \propto[-0.269,-0.269,0.925,0,0,0]^{T}, \\
& \overline{\sigma_{2}} \propto[0.654,0.654,0.381,0,0,0]^{T}, \\
& \overline{\sigma_{3}} \propto[-0.354,0.354,0,-0.703,0.505,0]^{T}, \\
& \overline{\sigma_{4}} \propto[0.612,-0.612,0,-0.407,0.292,0]^{T}, \\
& \overline{\sigma_{5}} \propto[0,0,0,-0.505,-0.703,-0.501]^{T}, \\
& \overline{\sigma_{6}} \propto[0,0,0,-0.292,-0.407,0.866]^{T} .
\end{aligned}
$$

(b) Calcite, $\mathrm{CaCO}_{3}$, has space group $R \overline{3} c$. Landolt-Börnstein (1979) gives its compliance constants in units of (TPa) $)^{-1}$ as $s_{11}=11.4, s_{33}=17.4, s_{44}=41.4, s_{12}=-4.0, s_{13}=-4.5, s_{14}=9.5$. Equation (15) then gives for the eigenvalues in $(\mathrm{TPa})^{-1}: \lambda_{1}=$ $20.5, \lambda_{2}=4.31, \lambda_{3}=27.9, \lambda_{4}=8.19$. The eigenangles $\omega=25.9^{\circ}$, $\xi=-37.2^{\circ}, \rho=0^{\circ}$ determine the eigenvectors:

$$
\begin{aligned}
& \overline{\sigma_{1}} \propto[-0.309,-0.309,0.899,0,0,0]^{T}, \\
& \overline{\sigma_{2}} \propto[0.636,0.636,0.437,0,0,0]^{T} \\
& \overline{\sigma_{3}} \propto[0.428,-0.428,0,0.796,0,0]^{T} \\
& \overline{\sigma_{4}} \propto[0.563,-0.563,0,-0.605,0,0]^{T} \\
& \overline{\sigma_{5}} \propto[0,0,0,0,0.796,0.605]^{T} \\
& \overline{\sigma_{6}} \propto[0,0,0,0,-0.605,0.796]^{T} ;
\end{aligned}
$$

$\rho=180^{\circ}$ does not give rise to eigenvectors.

\section{Stress and strain eigenvectors, inequalities for the compliance components of trigonal crystals}

Defining

$$
\boldsymbol{\sigma}=\sum_{i=1}^{6} \overline{\boldsymbol{\sigma}_{i}}=\left[\sigma_{1}, \sigma_{2}, \sigma_{3}, 2^{1 / 2} \sigma_{4}, 2^{1 / 2} \sigma_{5}, 2^{1 / 2} \sigma_{6}\right]^{T}
$$

and

$$
\boldsymbol{\varepsilon}=\left[\varepsilon_{1}, \varepsilon_{2}, \varepsilon_{3}, 2^{-1 / 2} \varepsilon_{4}, 2^{-1 / 2} \varepsilon_{5}, 2^{-1 / 2} \varepsilon_{6}\right]^{T},
$$

we can write (5) as

$$
\boldsymbol{\varepsilon}=\mathbf{S} \cdot \boldsymbol{\sigma}
$$

We write (16) as

$$
\overline{\boldsymbol{\sigma}_{i}}=\mathbf{E}_{i} \cdot \boldsymbol{\sigma}, \quad i=1, \ldots, 6 .
$$

The $\overline{\boldsymbol{\sigma}_{i}}$ being mutually orthogonal, for arbitrary $\boldsymbol{\sigma}$, the $\mathbf{E}_{i}, i=1$, $\ldots, 6$, are projection operators onto one-dimensional subspaces of the six-dimensional space of symmetric secondrank tensors. Being projection operators, the $\mathbf{E}_{i}$ are idempotent, i.e. $\mathbf{E}_{i}^{2}=\mathbf{E}_{i}$.

The $\overline{\boldsymbol{\sigma}_{i}}$ are the eigenstates of the compliance tensor $\mathbf{S}$, called stress eigenstates. Choosing appropriate solutions $\omega, \xi$ and $\rho$ of equations (18) and defining $\lambda_{5}=\lambda_{3}, \lambda_{6}=\lambda_{4}$, we obtain

$$
\boldsymbol{\varepsilon}=\mathbf{S} \cdot \boldsymbol{\sigma}=\lambda_{\mu} \overline{\boldsymbol{\sigma}_{\mu}}=\left(\lambda_{\mu} \mathbf{E}_{\mu}\right) \boldsymbol{\sigma},
$$

where summation from 1 to 6 over repeated Greek indices is understood.

Defining strain eigenstates $\overline{\boldsymbol{\varepsilon}_{i}}$ by

$$
\overline{\boldsymbol{\varepsilon}_{i}}=\lambda_{i} \overline{\sigma_{i}}, \quad i=1, \ldots, 6,
$$

the generalized Hooke law (5) is decomposed into six laws of proportionality between the eigenstates of stress and strain. These eigenstates satisfy for $i \neq j$ :

$$
\overline{\boldsymbol{\sigma}_{i}} \cdot \overline{\boldsymbol{\sigma}_{j}}=0, \quad \overline{\boldsymbol{\sigma}_{i}} \cdot \overline{\boldsymbol{\varepsilon}_{j}}=0, \quad \overline{\boldsymbol{\varepsilon}_{i}} \cdot \overline{\boldsymbol{\varepsilon}_{j}}=0 .
$$

Thermodynamic arguments require the strain matrix $c_{\mu \nu}$ to be positive definite (see e.g. Nye, 1985). It follows that also the inverse matrix is positive definite, i.e. the compliance matrix $s_{\mu \nu}$. Its eigenvalues (15) must therefore all be positive, from which it follows that

$$
\begin{gathered}
s_{11}>0, \quad s_{33}>0, \quad s_{44}>0, \quad s_{11}>\left|s_{12}\right|, \\
\left(s_{11}+s_{12}\right) s_{33}>2 s_{13}^{2}, \quad\left(s_{11}-s_{12}\right) s_{44}>2\left(s_{14}^{2}+s_{15}^{2}\right) .
\end{gathered}
$$

Notice that the last restriction in (26) is stronger than the last two restrictions in equations (37) of Theocaris \& Sokolis (2000b); the last two of their restrictions (37b) as well as (37c) follow from the other restrictions (37).

\section{The form of the linear elastic tensor for arbitrary point groups in three dimensions}

The form of the linear elastic tensor is of interest not only for the crystallographic point groups but also for the point groups of quasicrystals or for isotropic or transversely isotropic media. We therefore shall discuss it for all the point groups in three dimensions that are listed in Table 10.1.4.2 of Hahn \& Klapper (2002). The linear elastic tensor, being a polar tensor of even rank, is invariant under the inversion 1 . It follows that invariance under a rotation and invariance under the same rotation combined with the inversion lead to the same restriction on the form of the tensor. Our task is made easy by the result obtained by Hermann (1934) that a rotation of order $n$ leads to the same restrictions as a rotation of infinite order for all tensors of rank $<n$. Generalizing the usual choice of a standard Cartesian coordinate system also to non-crystal- 
lographic groups by choosing the principal rotation axis along $x_{3}$ and a possible rotation axis perpendicular to the principal one along $x_{1}$, we find that there are no other restrictions on the form of the compliance matrix $s_{\mu \nu}$ and the stiffness matrix $c_{\mu \nu}$ than those given in Table 9 of Nye (1985).

For hexagonal point groups, $s_{\mu \nu}$ has the form

$$
\left[\begin{array}{cccccc}
s_{11} & s_{12} & s_{13} & 0 & 0 & 0 \\
s_{12} & s_{11} & s_{13} & 0 & 0 & 0 \\
s_{13} & s_{13} & s_{33} & 0 & 0 & 0 \\
0 & 0 & 0 & s_{44} & 0 & 0 \\
0 & 0 & 0 & 0 & s_{44} & 0 \\
0 & 0 & 0 & 0 & 0 & s_{66}
\end{array}\right], \quad \text { where } s_{66}=2\left(s_{11}-s_{12}\right)
$$

The form of $c_{\mu \nu}$ is similar, except that $c_{66}=\frac{1}{2}\left(c_{11}-c_{12}\right)$. These forms are valid also for the $2 N$-gonal non-crystallographic groups $n, \bar{n}, n / m, n 22, n m m, \bar{n} 2 m, n / m m m$ with $n=2 N, N \geq 4$, the $(2 N+1)$-gonal non-crystallographic groups $n, \bar{n}, n 2, n m$, $\bar{n} m$ with $n=2 N+1, N \geq 2$, and the cylindric groups $\infty, \bar{\infty}$, $\infty 2, \infty m, \bar{\infty}$. The form (27) is also called transversely isotropic.

The isotropic form of $s_{\mu \nu}$ is

$$
\left[\begin{array}{cccccc}
s_{11} & s_{12} & s_{12} & 0 & 0 & 0 \\
s_{12} & s_{11} & s_{12} & 0 & 0 & 0 \\
s_{12} & s_{12} & s_{11} & 0 & 0 & 0 \\
0 & 0 & 0 & s_{44} & 0 & 0 \\
0 & 0 & 0 & 0 & s_{44} & 0 \\
0 & 0 & 0 & 0 & 0 & s_{44}
\end{array}\right], \quad \text { where } s_{44}=2\left(s_{11}-s_{12}\right)
$$

The form of $c_{\mu \nu}$ is similar, except that $c_{44}=\frac{1}{2}\left(c_{11}-c_{12}\right)$. These forms are valid also for the icosahedral groups $235, m \overline{3} \overline{5}$ and the spherical groups $2 \infty, m \bar{\infty}$.

These results can be summarized as follows: The compliance and stiffness tensors are transversely isotropic for all point groups with a unique rotation axis of order $n \geq 5$, and isotropic for all point groups with several rotation axes of order $n \geq 5$.

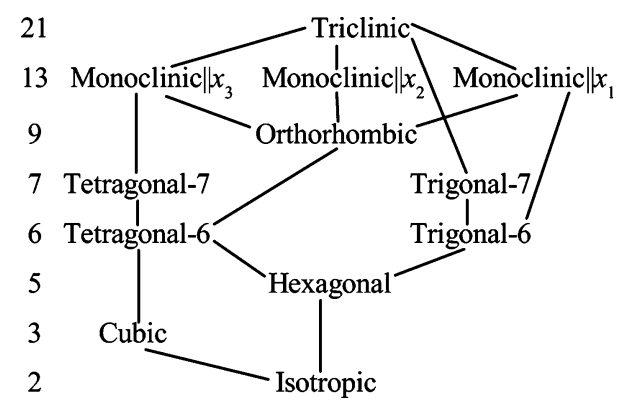

Figure 1

Restrictions on the first-order compliance and stiffness tensors for the standard choice of the Cartesian coordinate system. The restrictions are stronger for the point-group symmetries at the lower end of a line than for those at the upper end. The numbers on the left indicate the numbers of independent tensor (and matrix) components.
Fig. 1 tells us which restrictions in Table 9 of Nye (1985), to which monoclinic $\| x_{1}$ has been added, are stronger or less strong than others.

\section{Coordinate-independent types of symmetry restrictions}

Selecting one of the three coordinate systems indicated in Fig. 1 for monoclinic point groups, we are left with ten possible types of symmetry restrictions for the first-order elastic tensors. However, there are only eight coordinate-independent types of restrictions, as pointed out by Forte \& Vianello (1996). This is because the coordinate-independent restrictions are the same for the two tetragonal Laue classes as well as for the two trigonal ones. If a secondary symmetry direction in Laue class $\overline{3} m$ is chosen along $x_{1}$, we shall have $s_{15}=0$; if it is chosen along $x_{2}$, we shall have $s_{14}=0$. Also in Laue class $\overline{3}$, we could obtain $s_{15}=0$ (or $s_{14}=0$ ) by choosing $x_{1}$ in the plane perpendicular to $x_{3}$ in an appropriate direction, which depends on the elastic properties of the crystal. Similarly, $s_{16}=0$ if a secondary symmetry direction in Laue class $4 / \mathrm{mmm}$ is chosen along $x_{1}$. Also in Laue class $4 / m$, we could obtain $s_{16}=0$ by choosing $x_{1}$ in the plane perpendicular to $x_{3}$ in an appropriate direction. It follows that there are only eight coordinateindependent types of restrictions. Fig. 2 tells us which of these are stronger or less strong than others.

Notice the line between the cubic and trigonal cases, which is missing in Fig. 1 because none of the threefold axes of cubic symmetry is along $x_{3}$ for the standard choice of the Cartesian coordinate system.

\section{Conclusions}

The fact that the number of different eigenvalues and the number of eigenangles add up to the number of independent coefficients in the matrix $s_{\mu \nu}$, which Theocaris \& Sokolis (1999, $2000 a$ ) found for the monoclinic, orthorhombic, tetragonal-7, tetragonal-6, hexagonal, cubic and isotropic cases, holds also for trigonal-7 and trigonal-6.

Necessary and sufficient restrictions for the components of $s_{\mu \nu}$ to make this matrix positive definite have been derived for

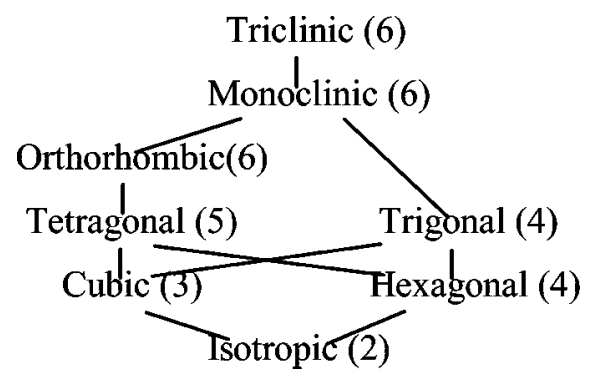

Figure 2

Coordinate-independent restrictions on the first-order compliance and stiffness tensors. The restrictions are stronger for the point-group symmetries at the lower end of a line than for those at the upper end. The numbers of independent eigenvalues of the corresponding matrices (5) and (11), respectively, are given in parentheses. 
the two trigonal cases, and it has been shown that the restrictions of Theocaris \& Sokolis $(2000 b)$ are not sufficient.

The hierarchy of restrictions on the linear elastic tensors that follow from Neumann's principle for arbitrary point groups in three dimensions has been established for the standard choice of the Cartesian coordinate system, as well as in coordinate-independent formulation.

\section{References}

Authier, A. \& Zarembovitch, A. (2003). International Tables for Crystallography, Vol. D, edited by A. Authier, pp. 72-98. Dordrecht: Kluwer Academic Publishers.

Forte, S. \& Vianello, M. (1996). J. Elasticity, 43, 81-108.

Hahn, Th. \& Klapper, H. (2002). International Tables for Crystallography, Vol. A, 5th ed., edited by Th. Hahn, pp. 761-808. Dordrecht: Kluwer Academic Publishers.
Hermann, C. (1934). Z. Kristallogr. 89, 32-48.

Humbert, P. \& Plicque, F. (1972). C. R. Acad. Sci. Paris Sér. B, 275, 391-394.

Landolt-Börnstein (1979). Numerical Data and Functional Relationships in Science and Technology. New Series, Group III: Crystal and Solid State Physics, Vol. 11, Elastic, Piezoelectric, Pyroelectric, Piezooptic, Electrooptic Constants, and Nonlinear Dielectric Susceptibilities of Crystals. Berlin: Springer.

Landolt-Börnstein (1984). Numerical Data and Functional Relationships in Science and Technology. New Series, Group III: Crystal and Solid State Physics, Vol. 18, Elastic, Piezoelectric, Pyroelectric, Piezooptic, Electrooptic Constants, and Nonlinear Dielectric Susceptibilities of Crystals. Berlin: Springer.

Nye, J. F. (1985). Physical Properties of Crystals: their Representation by Tensors and Matrices. Oxford: Clarendon Press.

Rychlewski, J. (1984a). PMM USSR, 48, 303-314.

Rychlewski, J. (1984b). Adv. Mech. 7(3), 51-80.

Theocaris, P. S. \& Sokolis, D. P. (1999). Acta Cryst. A55, 635-647.

Theocaris, P. S. \& Sokolis, D. P. (2000a). Acta Cryst. A56, 319-331.

Theocaris, P. S. \& Sokolis, D. P. (2000b). Z. Kristallogr. 215, 1-9.

Theocaris, P. S. \& Sokolis, D. P. (2001). Acta Mech. 150, 237-261. 